\title{
A Novel System for Elderly Fall Safety using Body Area Network
}

\author{
M. N. Nyan*; Francis E. H. Tay*, ; D. G. Guo*; L. Xu*; K. L. Yap* \\ *National University of Singapore, Department of Mechanical Engineering \\ ${ }^{+}$Institute of Bioengineering and Nanotechnology, Medical Devices Group \\ mpenmn@nus.edu.sg
}

\begin{abstract}
A system based on Body Area Network for detecting the onset of faint fall using motion sensors is presented. It is a portion of the project for elderly fall safety in which abnormal ECG and blood pressure vital signs are used to predict the imminent faint and fall onset is subsequently detected in activating the feedback systems. A statement of hypothesis, proof and experimental results are thoroughly discussed and presented.
\end{abstract}

Keywords: Body Area Network, elderly, fall.

\section{INTRODUCTION}

Falls and fall-related injuries are the most common causes of injury and hospitalization among the elderly [1]. Typically, fainting is one of the important causes of falls and its unexplained recurrence is common in the elderly as they grow older. Around $40 \%$ to $60 \%$ of faint-related falls are unwitnessed [2]. Such falls are a substantial public health problem because they are the primary cause of accidental deaths in people older than 65 years [3], and can result in fractures, commonly hip fractures, which cause morbidity and a decline in activities of daily living and in physical and social activities. Thus, a garment-based wearable faint fall safety system (MEMSWear) will offer great advantages to health care for the elderly in terms of helping those with fall experiences regain confidence or living independently and reduce elderly health care expenditure. The system is competent in (1) predicting an imminent faint using blood pressure and ECG vital signs and (2) subsequent detection of the fall onset using MEMS motion sensors such as accelerometers and gyroscopes (Fig. 1). Once fall is confirmed at its onset stage, the system will activate the equipped fall prevention and injury minimization units before the wearer touches the ground. For the comfort of the wearer in long term application, the whole system is based on the Body Area Network (BAN) (Fig. 1). In this paper, the novel strategic approach of faint fall onset detection using motion sensors will be discussed.

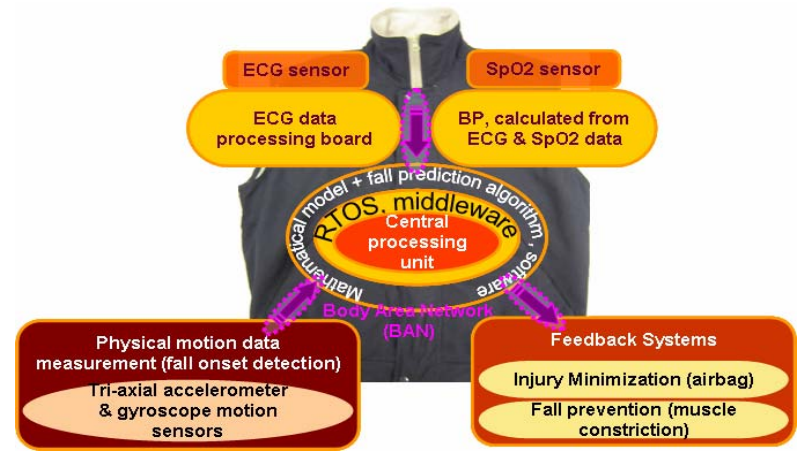

Fig. 1. BAN-based elderly fall safety system

Permission to make digital or hard copies of all or part of this work for personal or classroom use is granted without fee provided that copies are not made or distributed for profit or commercial advantage and that copies bear this notice and the full citation on the first page. To copy otherwise, to republish, to post on servers or to redistribute to lists, requires prior specific permission and/or a fee.

BODYNETS 2007, June 11-13, Florence, Italy

Copyright ( 2007 ICST 978-963-06-2193-9

DOI 10.4108/bodynets.2007.157

\section{METHODOLOGY}

The statement that "thigh segment does not go beyond certain threshold angle in forward and sideways directions in normal activities and this abnormal behavior occurs in faint falls" is hypothesized in onset detection. In proof of the statement, a hardware setup was developed. Thigh sensor set (TS) contains one MMA7260Q $( \pm 4 \mathrm{~g}, 300 \mathrm{mV} / \mathrm{g})$ tri-axial micromachined accelerometer $\{x$ : vertical (downward positive); $y$ : lateral (right positive); z: sagittal (forward positive) $\}$ and two ADXRS150 $( \pm 150 \%$ sec) yaw rate gyroscopes measuring in lateral (left positive) and sagittal (back positive) directions. Only one tri-axial accelerometer is included in waist sensor set (WS) with similar sensitivity axis setting as that in TS. TS is attached using Velcro at the front, slightly above mid level, of one thigh. WS is attached on a belt at front waist position. Chipcon CC2420 Zigbee transceivers were used for data communication between sensor sets and central processing unit located on MEMSWear Smartshirt. A Zigbee System-on-Chip (SoC), CC2431 by Chipcon is deployed as the central processing unit. Sensor data is sampled at sampling rate of $47 \mathrm{samples} / \mathrm{sec}$.

Fig. 2 outlines the process flow of the onset detection algorithm. $i^{\text {th }}$ sample of accelerometers $\left(x_{T S}(i), y_{T S}(i)\right.$, $\left.{ }_{T S}(i), x_{W S}(i), y_{W S}(i), z_{W S}(i)\right)$ are low pass filtered with cutoff frequency less than and equal to $0.5 \mathrm{~Hz}$ and those of gyroscopes $\left(\omega_{L A T}(i), \omega_{S A G}(i)\right)$ are band pass filtered with pass band between $0.01 \mathrm{~Hz}$ and $2.5 \mathrm{~Hz}$. Then, acceleration samples are transformed into a two dimensional measurement of body orientation, measuring how many degrees these body segments, thigh segment and trunk segment, have deviated from the vertical axis, e.g., standing is zero degree and lying flat on the floor is ninety degree, using the following equations,

$$
\begin{aligned}
& \operatorname{deg}_{S A G}=-\operatorname{atan}\left(z_{T S o r W S} / x_{T S o r W S}\right) *(180 / p i), \\
& e=\operatorname{sqrt}\left(1-\left(y_{T S o r W S} * y_{T S o r W S}\right)\right), \text { and } \\
& \operatorname{deg}_{L A T}=\operatorname{atan}\left(y_{\text {TSorWS }} / e\right) *(180 / p i) .
\end{aligned}
$$

$\operatorname{deg}_{S A G}$ represents rotational degrees in sagittal plane and $\operatorname{deg}_{L A T}$ represents those in lateral plane. If $\operatorname{deg}_{S A G}$ (positive for backward rotation) or $\operatorname{deg}_{L A T}$ (positive for left side rotation) hits the threshold value $\pm 10 \mathrm{deg}$, two steps of fall confirmation are processed. The first step is calculating the correlation coefficient ( $\rho_{\text {deg }} \geq 0.8$ ) of two angle data segments $\left\{\operatorname{deg}_{T S}(-N+i) ; i=0, \ldots, N\right.$ and $\left.N=19\right\}$ and $\left\{\operatorname{deg}_{W S}(-N+i) ; i=0, \ldots, N\right.$ and $\left.N,=19\right\}$ where $\operatorname{deg}(0)$ 
is detected point of $\pm 10 \mathrm{deg}$, from two sensor sets. The second step applies correlation coefficient ( $\rho_{\omega} \geq 0.88$ )

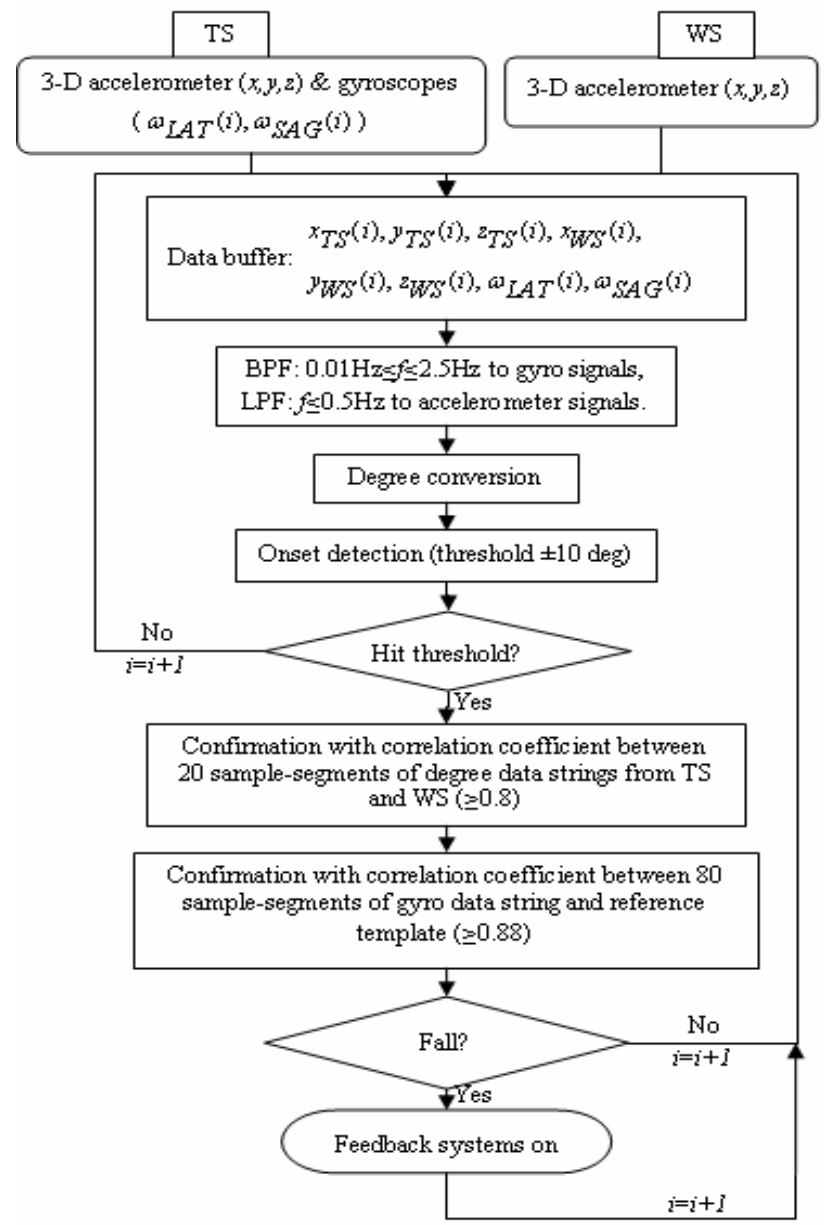

Fig. 2. Flow chart of fall onset detection system

between the band pass filtered gyroscope segment $\{\omega(-N+i) ; i=0, \ldots, N$ and $N=79\}$, where $\omega(0)$ is detected point of $\pm 10 \mathrm{deg}$, and its corresponding reference template. Here, the word "corresponding" means, the detected positive lateral/sagittal degree sample implies correlation of the gyro-segment with left/backward fall reference template while the detected negative lateral/sagittal degree sample involves correlation with right/forward fall reference template. This is because of gyroscope sensors' sensitivity orientations measuring in lateral (sensitivity axis: left positive) and sagittal (sensitivity axis: back positive) plains. Feedback systems will be switched on if the coefficients are above or equal to 0.8 and 0.88 . In this detection, only the thigh degree samples traveling from 0 to \pm 90 degrees, as thigh shifts from 0 to \pm 90 degrees in fall activities, are taken as points of interest for confirmation process. That can be chosen by measuring the slope of the sample as $\left(\left(\operatorname{deg}_{i}-\operatorname{deg}_{i-5}\right) / 5\right)$, where $\operatorname{deg}_{i}$ is equal to \pm 10 degree. Then, $i^{\text {th }}$ sample is changed to $(i+1)^{\text {th }}$ sample in the data buffer and process is looping till the system is switched off. The whole algorithm for $i^{\text {th }}$ samples are completed within $21 \mathrm{msec}$ sampling interval before next $i+1^{\text {th }}$ sample is taken.
In summary, the hypothesis that "thigh segment goes beyond certain threshold angle in forward and sideways directions only in falls, not in normal activities" is proved using the algorithm and hardware setting stated above.

\section{RESULTS}

Thirteen male and eight female volunteers, year fourth medical students from Singapore General Hospital, participated in the testing. The average ages were 23.38 and 22.25 years old, respectively, with a range of 22 to 27 for males and 21 to 23 for females. The mean height and mass \pm standard deviation of the males were $1.758 \pm 0.0557 \mathrm{~m}$ and $69.1 \pm 8.72 \mathrm{~kg}$, respectively. For the female volunteers, the mean height \pm standard deviation was $1.648 \pm 0.0282 \mathrm{~m}$, and the mean mass \pm standard deviation was $51.58 \pm 4.468 \mathrm{~kg}$. Informed consents were obtained from each of the subjects. In faint fall simulation, the subjects were told to stand by the mattress, relaxed themselves and fall to the sides, back and front. The subjects performed the simulated fainting incidents on a 6-inch thick soft foam mattress. For normal activities, a chair, the mattress and two flights of stairs were used for sitting, sit-stand transition, walking, stand-sit transition, lying, ascending and descending stairs. These normal activities are assumed as basic daily activities among the elderly. Each activity was conducted twice and recorded using a camcorder. The observer reset the two systems, camcorder and wearable system, at the same time in the experiment. Five arbitrary data sets of falls were used to develop coefficient threshold values and reference templates. The rest were used in validation.
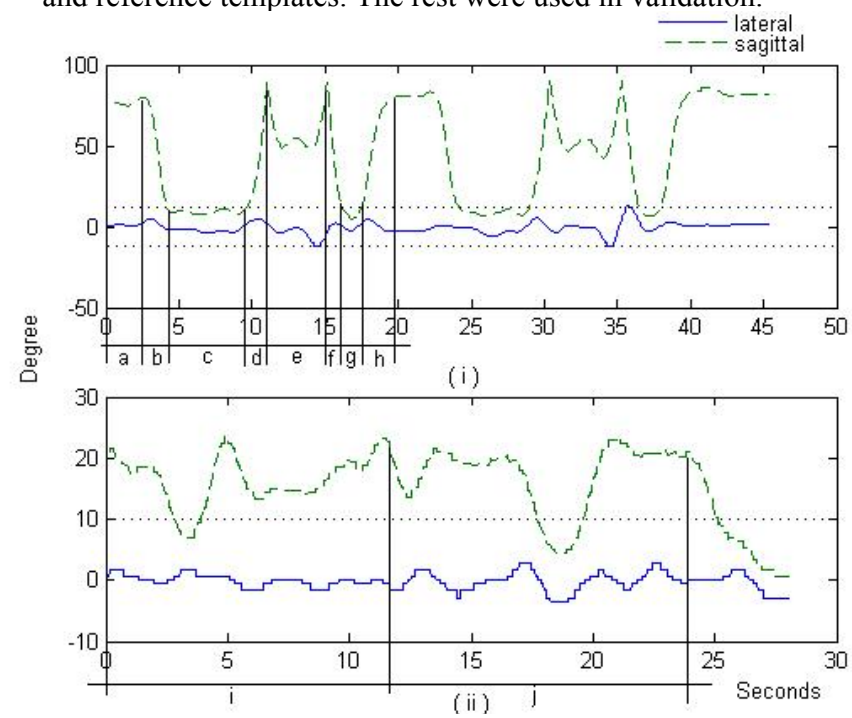

(a) sitting position (sitting on the chair), (b) sit-stand transition, (c) walking, (d) stand-sit transition (sitting on the mattress), (e) sit-lie-sit, (f) sit-stand transition, (g) walking, (h) sitting on the chair, (i) ascending stairs and (j) descending stairs.

\section{Fig. 3. Degree of thigh segment shifted from vertical position in lateral and sagittal directions for normal activities.}

Since equations 1,2 and 3 convert thigh and trunk segments' three dimensional acceleration signals to rotational angle information, zero degree in vertical position and 90 
degrees in horizontal position, thigh segment's sagittal angles (Fig. 3) shows approximately ninety degrees in sitting and lying activities, approximately zero degree in walking and standing activities and zero to ninety and ninety to zero degree transitions in sit-stand and stand-sit transition activities. In walking on stairs activities, it varied between zero and twenty degrees. It is obvious that movement is more prominent in sagittal plain than in lateral plain in normal activities. In support of the hypothesis, Fig. 3(i, ii) shows no intersections between -10 degrees threshold levels and sagittal degree curves (related to forward fall). For lateral degree curves (related to sideways falls), there are only three momentary points of contacts with \pm 10 degrees levels in Fig. 3(i) and no intersections at all in Fig. 3(ii). This can be interpreted as "thigh segment does not go beyond certain threshold angle in forward and sideways directions in normal activities". These three momentary contact points can be avoided by setting higher threshold levels, but it can result in shorter lead time, the time interval from the time when fall onset is detected ( \pm 10 degrees) to end of the fall. End of the fall is decided from the video clips in which it is the point when the subject comes in contact with the mattress. Stand-sit transitions, similar to backward falls, are successfully rejected using $\rho_{d e g}$ as opposite movements of thigh segment and trunk segment during stand-sit transition results in negative $\rho_{\text {deg }}$.
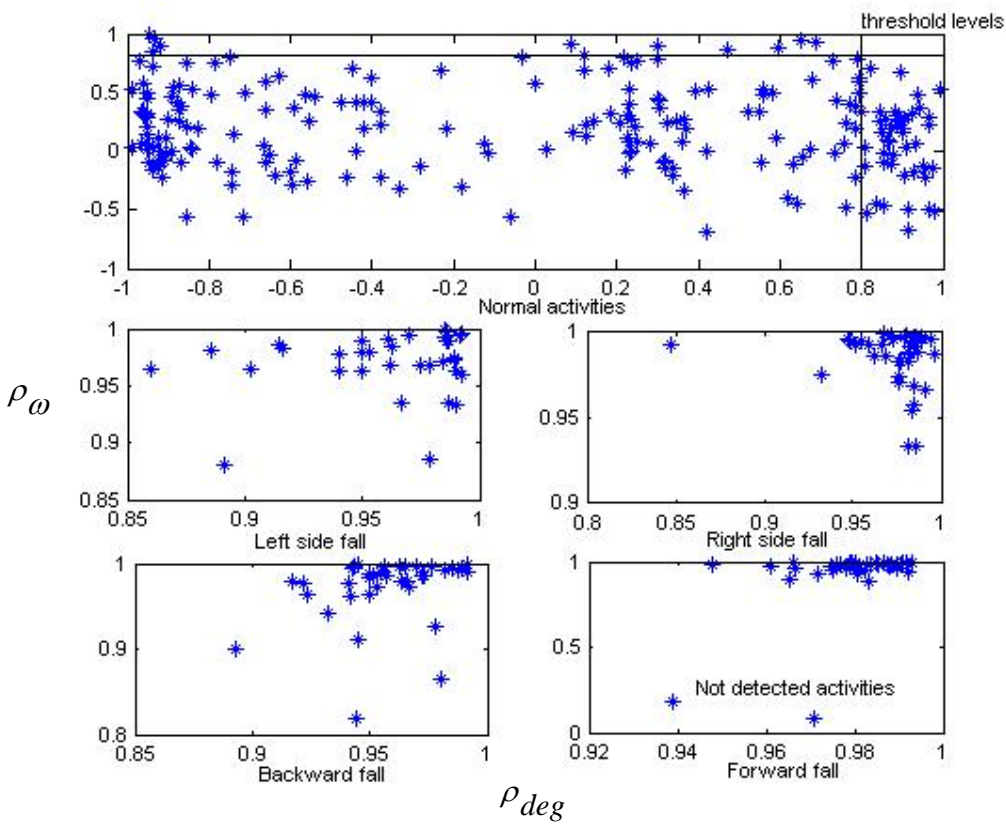

Fig. 4. $\rho_{\mathrm{deg}}$ and $\rho_{\omega}$ relationships of falls and normal activities

Only two in thirty seven front falls were not detected resulting in 94.6 percent sensitivity for forward fall, while 100 percent sensitivity for other falls with no false alarms, where sensitivity was computed as activities detected (35 forward falls) divided by total activities ( 37 forward falls used in validation). The reason of the failure with these two cases was that two ladies conducted kneeling down first before they release their bodies onto the mattress to forward direction. In kneeling down, thigh go backward while trunk is moved forward and the correlation coefficients of gyroscope segments, $\rho_{\omega}$, become lower than the threshold value. Actually, this way of falling is not typical in unconscious faint fall that usually cause major injuries. Two hundred and sixteen data sets of normal activities were plotted and this figure was the number of detected points using \pm 10 degree threshold levels for all twenty one subjects. Thirty seven data sets, $42-5=37$ sets, were plotted for fall activities. Relationships of correlation coefficients between $\rho_{\text {deg }}$ and $\rho_{\omega}$ for falls, 37 sets, and normal activities, 216 sets, are shown in Fig. 4. Correlation coefficients, $\rho_{d e g}$ and $\rho_{\omega}$, are smaller than 0.8 and 0.88 threshold values in normal activities but are higher than those values in fall activities. Means and standard deviations of lead times for fall activities, 37 sets, are shown in Fig. 5 and these values exist between 0.6 second and 0.8 second.

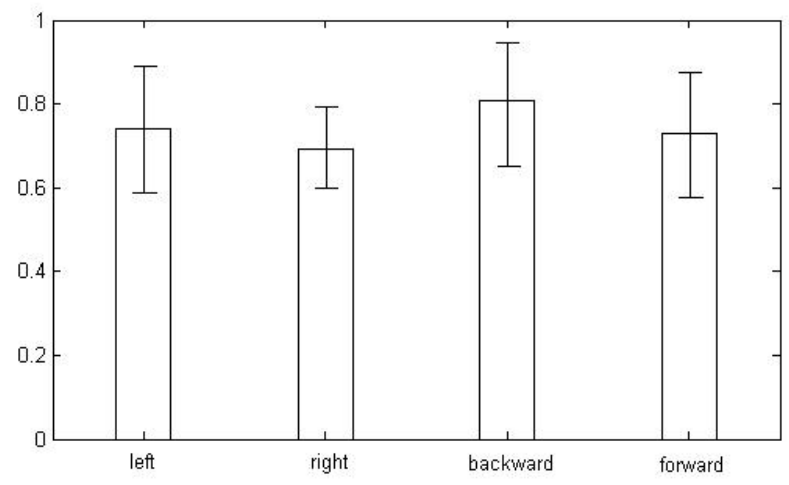

Fig. 5. Means and standard deviations of lead times (seconds) for fall activities

\section{CONCLUSIONS}

The theme discussed in this paper is a part of the project aimed at developing a wearable fall safety system for the elderly. Through MEMS and Zigbee wireless data transmission as the enabling and state of the art technologies, the development of a reliable, light weight, and smart wearable system with low power consumption and maximum comfort in long-term application is possible and enhances the standard of health care services.

\section{ACKNOWLEDGEMENT}

The project is one of those under Embedded \& Hybrid Systems Phase II Programme funded by Science \& Engineering Research Council (SERC), Singapore.

\section{REFERENCES}

[1] Kannus, P., Sievänen, H., Palvanen, M., Järvinen, T., Parkkari, J. Prevention of falls and consequent injuries in elderly. The Lancet, 366, 9500 (Nov. 2005- Dec. 2005), 1885-1893.

[2] McIntosh, S., Da Costa, D., Kenny, R. A. Outcome of an integrated approach to the investigation of dizziness falls and syncope in elderly patients referred to a 'syncope' clinic. Age Ageing, 22 (1993), 53-8.

[3] Liebenstein, H. A theory of economic development. Princeton: Princeton University Press, 1954. 